
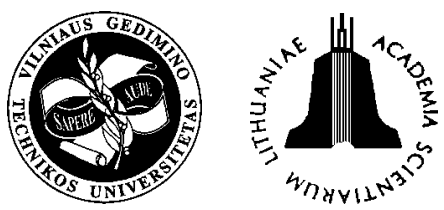

ISSN 1648-4142 TRANSPORT

http:/www.vtu.lt/english/editions

\title{
DYNAMICS OF PNEUMATIC AND HYDRAULIC TRANSPORT TECHNOLOGICAL EQUIPMENT
}

\author{
Marijonas Bogdevičius, Arvydas Matuliauskas, Vygantas Mištinas, Bronislovas Spruogis, \\ Vladimiras Suslavičius \\ Dept of Transport Technologic Equipment, Vilnius Gediminas Technical University, \\ Plytinès g. 27, LT-10105 Vilnius-16, Lithuania. E-mail: tti@ti.vtu.lt, tel.: (+370 5) 2744783, fax: (+370 5) 2745060
}

Received 2003-11-26; accepted 2004-01-15

\begin{abstract}
In the article the classification of inpipe robots is presented, their advantages and imperfections are reviewed. The schemes of the construction of the wall press walking inpipe robot with a pneumatic drive and an inpipe robot with a vibratory pneumatic drive are given. The analysis of the identification method of leaks occurring due to damages of the linear part of the main oil pipelines is carried out. The momentum and continuity equations of viscous compressible fluid in a pressure pipeline are presented. Differential equations of fluid movement in the oil pipeline are solved by the method of characteristics. Variation diagrams of pressure and velocity of fluid in a leaking oil pipeline are presented. The impulsive extinguishing systems are presented.
\end{abstract}

Keywords: inpipe robot, robot with a pneumatic drive, oil pipeline, damage, identification, numerical method, wave speed, impulsive extinguishing technologies.

\section{Introduction}

Pipelines are major tools for the transportation of fluids and gases and a number of countries employ pipelines as the main facilities. Recently, there is a lot of troubles caused by aging, corrosion, cracks, and mechanical damages from third parties. As a result in pipelines pressure decreases and wastes increase. Therefore the diagnostics of pipelines defects is a topical problem.

\section{The classification of inpipe robots}

Continuous activities for inspection, maintenance and repair are strongly demanded. However, these activities need enormous budgets that may not be easily handled by related industries. For these reasons, the application of the robot for the maintenance of the pipelines appears to be one of the most attractive solutions to be delivered now. Inpipe robots can be classified into several elementary [1-3] forms according to the locomotion mechanism as shown in Fig 1. Most of them have been designed based on specific tasks. As shown in Fig 1(a), for example, the pig is one of the most well known commercial one that is passively driven by fluid pressure inside oil pipelines and employed for the inspection of pipelines with large diameters. The wheel type illustrated in Fig 1(b) is similar to the plain mobile robot and a number of commercialized can be found.

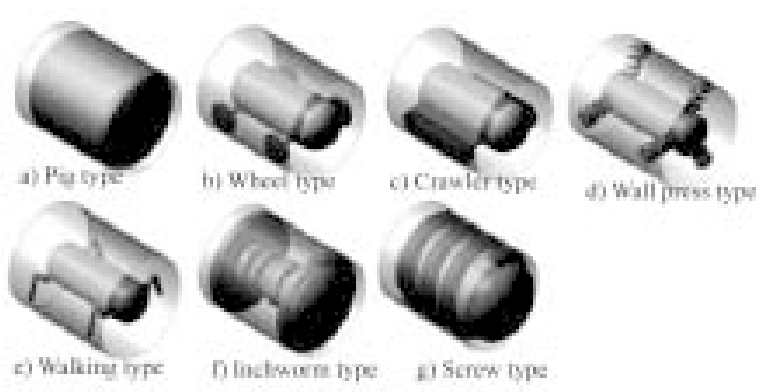

Fig 1. Classification of inpipe robots

The advantages of this group of design of inpipe robots are the following: self-propelling; good control of the speed of movement and the force of cohesion with the contact surface; good capacity of piping where diameters of pipes are different; sufficient capacity in bent fragments of piping.

The imperfections of this group of design of inpipe robots are the following: they can be used only after the termination of supply of the transported substance; limited possibilities of power supply; rotational movement on the contact with the pipe.

Fig 1(c) shows a crawler type robot with caterpillars instead of wheels. As shown in Fig 1(d), a wall press type denotes the robot with flexible mechanism for pressing the wall whatever means that it has an advantage of climbing vertical pipelines. As depicted in Fig 1(e), a 
walking type possesses articulated legs and it can produce various motions.

The advantages of these groups of design of inpipe robots are the following: self-propelling; no motion at the contact with the pipe; good control of the speed of movement and the force of cohesion with the contact surface.

The imperfections of this group of construction of inpipe robots are the following: they can be used only after the termination of supply of the transported substance; limited possibilities of power supply; limited movement when the layer of coating on the inner surface of the pipe is too thick; limited capacity in bent fragments of piping; limited capacity of piping with different diameters of pipes.

The inchworm type given in Fig 1(f) is usually being developed for pipelines with small diameter. Screw type one takes the motion of a screw when it advances in the pipelines as depicted in Fig 1(g). Most of inpipe robots take the mechanism derived from one of those basic mechanisms or their combinations [1-5]. The goals of the inpipe robot have close relations with the task space of specific applications. The principal requirement of the inpipe robot is that the robot has to explore anywhere it would go within its task space. Existing robots generally travel along horizontal pipelines smoothly, but some of them move along vertical pipelines or elbows (bend pipes, L-shaped pipes). Furthermore, a small minority of them can achieve the selective navigation in either common type of branches (T-shaped pipes) or branches with the radical radius of $1,5 \mathrm{D}$ curvature. For effective inspection, however, inpipe robots should have the ability to negotiate elbows and branches, since there exists a number of those special fittings in pipelines, especially urban gas pipelines.

In the department of transport technological equipment two inpipe transport robots with pneumatics drives were made. They can be classified to transport diagnose devices of inpipe surfaces defects.

One of them, a wall press walking inpipe robot with a pneumatic drive, can be classified to a mixed type of a wall press type as shown in Fig 1(d) and a walking type as depicted in Fig 1(e).

This robot consists of few main elements (Fig 2) compressor 1 supplying pressured air to system, robots drive 2 , operating block 3 , pipes 4 which connect elements of control block network [4].

\subsection{Construction working principle of the wall} press walking inpipe robot with a pneumatic drive

A drive of the wall press walking inpipe robot with a pneumatic drive is illustrated in Fig 3. Robots drive consists of two locking blocks 1 , which can turn by hinges 2 small angles one in point of the other and intercon-

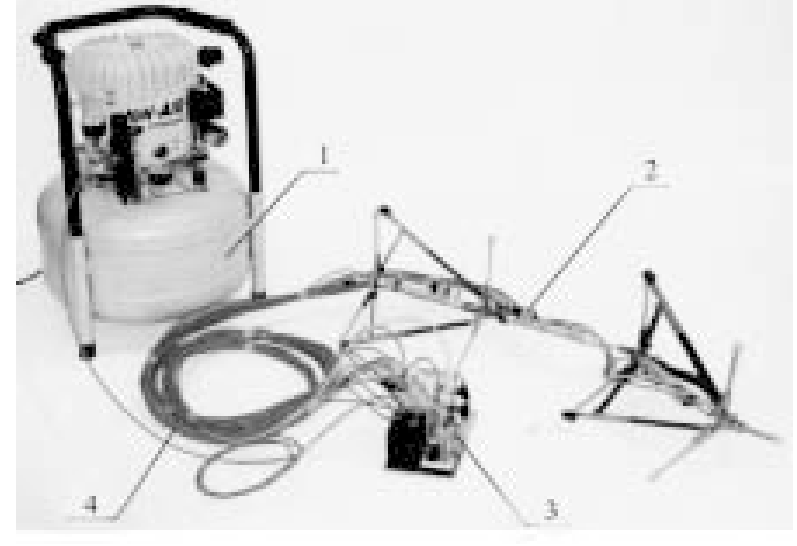

Fig 2. The scheme of the wall press walking inpipe robot with a pneumatic drive: 1 - compressor, 2 - robots drive, 3 - operating block, 4 - pipes

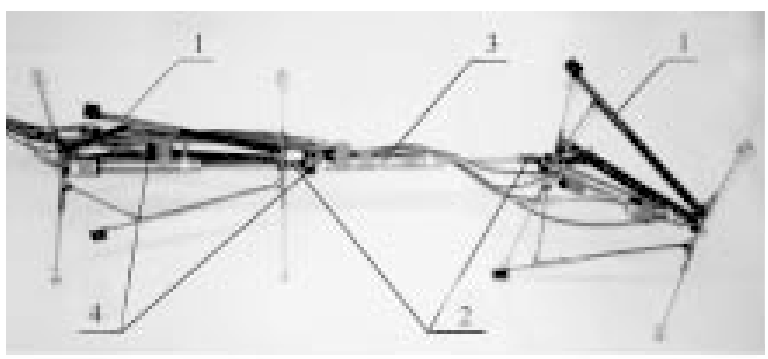

Fig 3. A drive scheme of the wall press walking inpipe robot with a pneumatic drive: 1 - locking block, 2 - hinge, 3 - shift pneumatic cylinder, 4 - operating sensors

nected, and propelled by a two-way working pneumatic cylinder 3.

As on a moving pneumatic cylinder as on locking blocks pneumatic cylinders pneumatic-magnetic operating sensors 4 are fixed. They send pneumatic signals to an operating block and process in operating block signals feeding cylinders of a pneumatic drive.

The principle of robot working is based on a walking movement, periodically pressing the wall of a pipeline with supporting elements of a locking block. Pressured air gets into a two-way working cylinder of a locking block and its drawing stock in contact between supporting elements and a pipeline wall fix a locking block. When one of two locking blocks is fixed, a pneumatic cylinder of other locking block, works in a reverse way. Supporting elements of the second locking block are released by a going out stock of a cylinder. Depending on which locking block contacts with a pipeline inside wall, a stock of a shift pneumatic cylinder goes out or goes in. In this way the wall press walking inpipe robot makes a move forward.

During a physical experiment of this inpipe robot the following dynamical parameters shown in Fig 4 were obtained. 


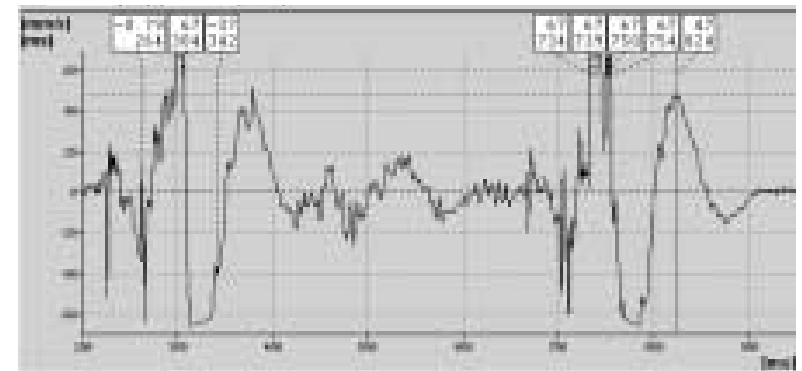

Fig 4. The velocity of the wall press walking inpipe robot with a pneumatic drive according to time

\subsection{Construction working principle of an inpipe} robot with a vibratory pneumatic drive

An inpipe inspection robot with a pneumatic drive consists of two segments with autonomous feeding. Passive modules such as a control module and other optional modules are inserted between the active vehicles [5]. All of the segments are interconnected with ruled elastic hinge joints, and in addition, the body of each section is fixed to one-stage elastic hinge dampers with 120r angle between them that bend at a certain angle with the direction opposite to the one of the movement; an elastic element is fixed between the said dampers and the side surface of the body.

The scheme of the segment of an inpipe inspection robot with a pneumatic drive is presented in Fig 5.

An inpipe inspection robot with a pneumatic drive consists of $n$ sections interconnected with ruled elastic hinge joints. Each section consists of the body 1 with three one-stage elastic hinge dampers 2 with $120^{\circ}$ angle between them fixed to it that are bent at the angle a against the direction opposite to the one of the movement. No less than two rows of such dampers must be provided.

In order to ensure the undirectional movement of the robot between the body 1 and the inner surface of the piping system as well as the contact surface of the dampers 2, a roll is mounted on the end of the damper. In the end part of the body the elastic element 4 is fixed and the piston 5 is fixed to its other end.

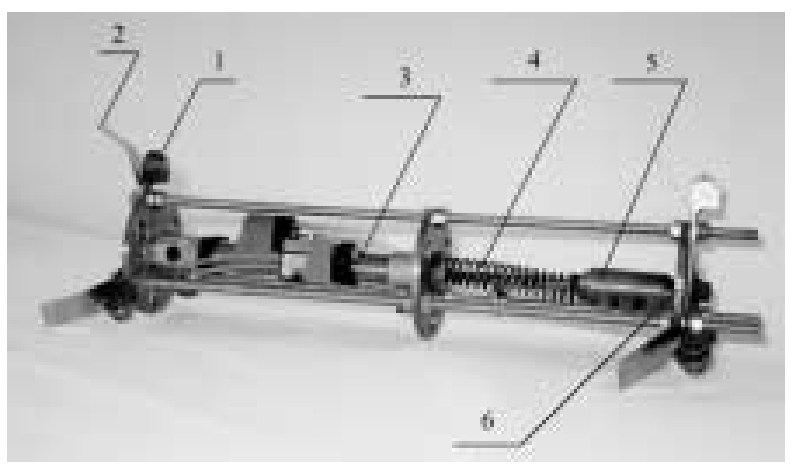

Fig 5. The design scheme of the original step movement inpipe inspection robot with a vibratory pneumatic drive: $1,2,4$ - the elastic element, 3 - the movement support, 5 - the piston, 6 - the damper
The compressed air is supplied from the pneumatic system receiver via the feeding branch pipe that causes vibrations of the piston 5 through the elastic element 4 . Therefore, the piston 5 contacts with the elastic element 3 fixed in the damper 2 and the element enables the dampers to be elastic and to change the trajectory of the movement in the piping, if the inner diameter of the piping as well as the thickness of the coating of the inner surface of the piping varies.

In order to ensure a reliable movement of the robot along the inner surface of the piping system and in order to reduce the friction on the contact surface between the inner surface of the piping system and the contact surface of the dampers 2, a roll may be mounted on the end part of the damper. In the end part of the body the elastic element 4 is fixed while the other end is connected to piston 5 . The branch pipes are provided in the end and side parts of the body for air supply and release.

During a physical experiment with this inpipe robot the following dynamical parameters of the system were obtained (See Fig 6). Authors thank KTU prof. $\mathrm{V}$. Barzdaitis for the equipment and help during the physical experiment.

\section{The method of leak identification in a pipeline}

Since the proportion of the fluid transported through these connections is rather small, the identification of leaks is very complicated. For fast detection of places of outflow of a liquid in the main pipelines the most effective is the method of impact wave that takes into account waves of pressure in a liquid in the main pipelines. On the basis of this method the method and the software of identification of places of outflow of a liquid in the main pipelines are developed.

The flow of viscous and compressive fluid is described by differential equations with partial derivatives, which express the laws of mass movement, quantity and conservation of energy.

Movement and continuity equations of viscous, compressible fluid in pressure pipe have the following form [6-14].

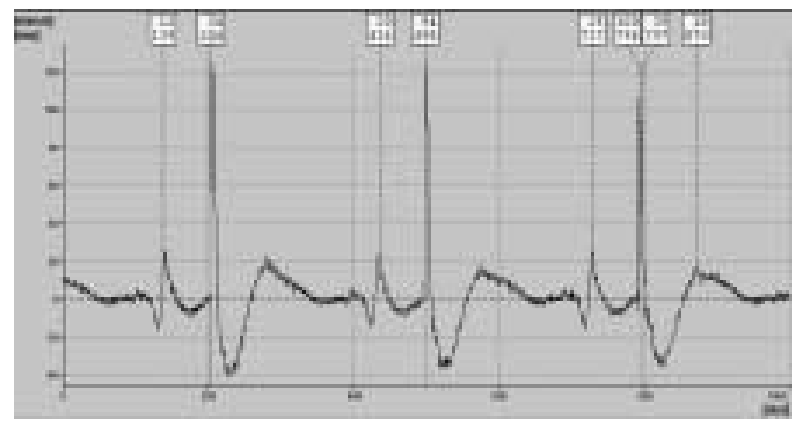

Fig 6. The velocity of an inpipe robot with a vibratory pneumatic drive during to time 


$$
\begin{aligned}
& \frac{1}{\rho} \frac{\partial p}{\partial x}+\frac{\partial v}{\partial t}+v \frac{\partial v}{\partial x}+\frac{f v|v|}{2 d}+a_{x}=0 \\
& \frac{\partial p}{\partial t}+v \frac{\partial p}{\partial x}+c^{2} \rho \frac{\partial v}{\partial x}=0,
\end{aligned}
$$

here: $p, v$ - pressure and velocity of fluid; $\rho$ - fluid density; $c$-velocity of sound; $f$-non-dimensional hydraulic coefficient of friction.

A crack in a pipeline causes rapid variations of fluid flow. The wave of rapidly reduced pressure propagates on both sides from the location of the crack at the speed of sound. Pressure and velocity vary rapidly when leakage occurs in a pipeline. Following the diagrams of pressure and velocity variations, fluid leak can be identified.

The method of leak identification is based on the observation of pressure wave propagation time when the change of pressure is registered. The identification of a leak is registered by the variation of time $\Delta t$ between pressure waves propagating into opposite sides from the leak that reach the beginning and the end of a pipeline, i.e. pressure sensing elements in the pipeline section under control, at different time. If leakage occurs in the middle of a pipeline section under control, variation of time $\Delta t$ will be equal to zero. Meanwhile, when leakage occurs closer to any end of the pipeline section under control, the variation of time $\Delta t$ of pressure wave propagation occurs. A sample diagram of pressure variation is presented in Fig 7 [7, 8].

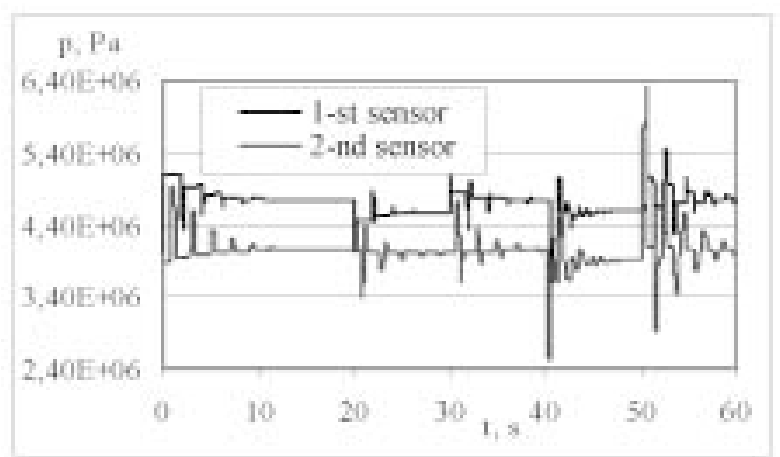

a)

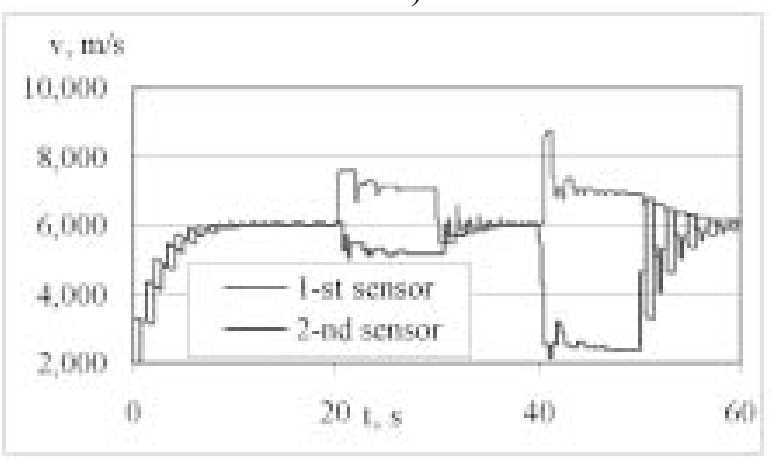

b)

Fig 7. Pressure and velocity variation diagram when the diameter of the first leaking pipe is $50 \mathrm{~mm}$, and the second $75 \mathrm{~mm}$ : a - pressure; $\mathrm{b}$ - velocity
Data and calculation results when the leak is identified according to the variations of velocity when diameters of leaking pipes are different (the length of a pipeline section is $1 \mathrm{~km}$ ) are illustrated in Fig 8 and Fig 9.

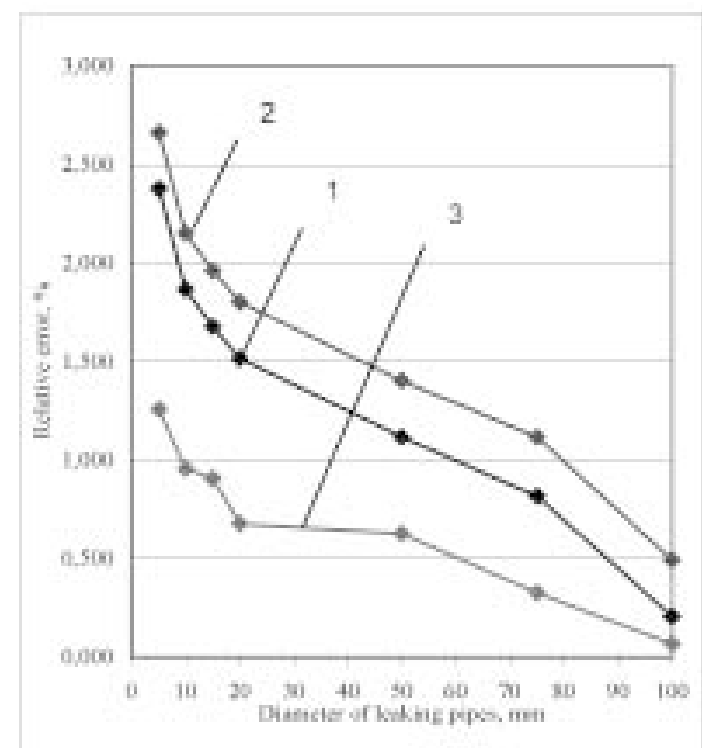

Fig 8. Dependences of relative errors on the diameter of a leaking pipe when the length of a pipeline section is $1 \mathrm{~km}$ 1 - calculation on pressure and taking account velocity of liquid; 2 - calculating on pressure and not taking into account velocity of liquid; 3 - calculation on liquid

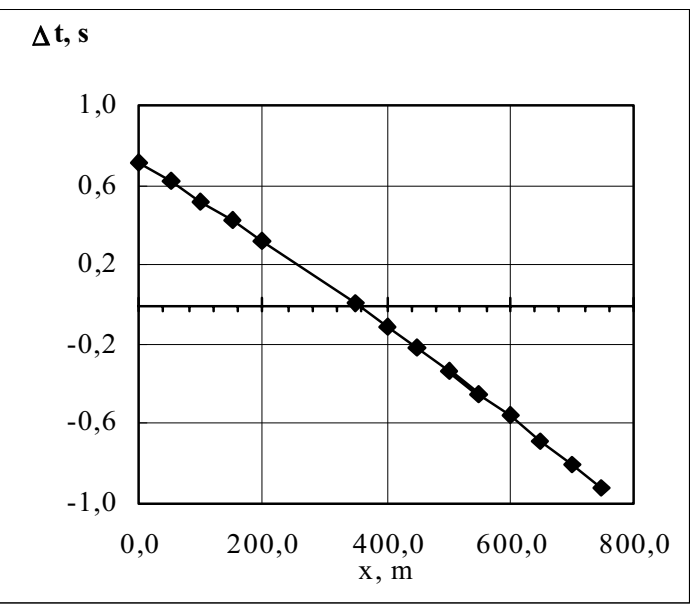

Fig 9. Graph of dependence $\Delta t=f(x)$ for $1 \mathrm{~km}$ long pipeline section ( $\Delta t-$ variation of time of waves propagating to the beginning and the end of the pipeline section under control)

\section{Deficiencies of the impulsive extinguishing technolo- gies}

Today water is chiefly supplied for extinguishing via volumetric and centrifugal pumps using its hydraulic liquid characteristics. The pressure energy of the pressurized and out-flowing water through the opening (i.e. fire nozzle) is transformed into jet kinetic energy. If we 
use the energy of the compressed air or other gases to eject water through the nozzle (instead of the compressed water energy) the jet speed could be much faster. The water droplet speed within the jet sprayed out in an ordinary way reaches tens $\mathrm{m} / \mathrm{s}$, while using the compressed air energy the water droplet speed can reach hundreds $\mathrm{m} / \mathrm{s}$. Furthermore, because of such speeds, water spray is atomized into fine droplets due to the air resistance (even up to 2 microns in diameter). Consequently, the covered area the extinguishing water enlarges as well as the water efficiency. In the table and Fig10 you can see droplet sizes, their number as well as the obtained covered area when water volume is one 1 liter [14].

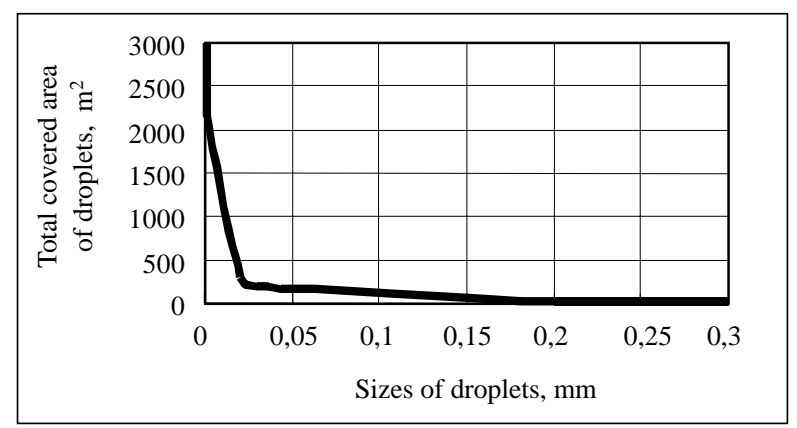

Fig 10. Dependence of the total covered area of 1 liter of water droplets on their diameter size

Diameters of water droplets, their number and the total surface area obtained from 1 liter of water

\begin{tabular}{|c|c|c|}
\hline $\begin{array}{c}\text { Droplet } \\
\text { diameters, } \mathrm{mm}\end{array}$ & Droplet number & Total surface, $\mathrm{m}^{2}$ \\
\hline 0,3 & $7,1 \times 10^{7}$ & 20 \\
0,2 & $2,4 \times 10^{8}$ & 30 \\
0,03 & $7,1 \times 10^{10}$ & 200 \\
0,02 & $2,4 \times 10^{11}$ & 300 \\
0,003 & $7,1 \times 10^{13}$ & 2000 \\
0,002 & $2,4 \times 10^{14}$ & 3000 \\
\hline
\end{tabular}

When water is supplied in fine droplets, it is possible to reach the use of all of its properties as close as $100 \%$. Impulsive nozzles function in the following order:

- Compressed air compartment is filled up from the air container;

- Water compartment is filled up from the water tank;

- When the fast reaction valve is opened, compressed air and water compartments get merged;

- Water being under air pressure is ejected in a very short time (from several to several tens of mili-seconds) to the focus of fire; ning.

- Further on the process is repeated from the begin-

Studies on the impulsive extinguishing technologies have not been completed yet and need to be further up- dated and tested. However, even now they allow us to use the properties of the extinguishing water more efficiently and reduce the response time.

The process of spraying water droplets from impulsive pneumatic and hydraulic system is shown in Fig 11.

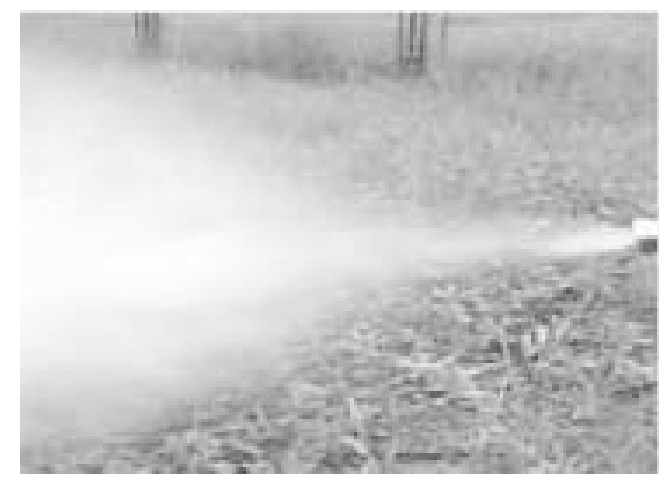

a)

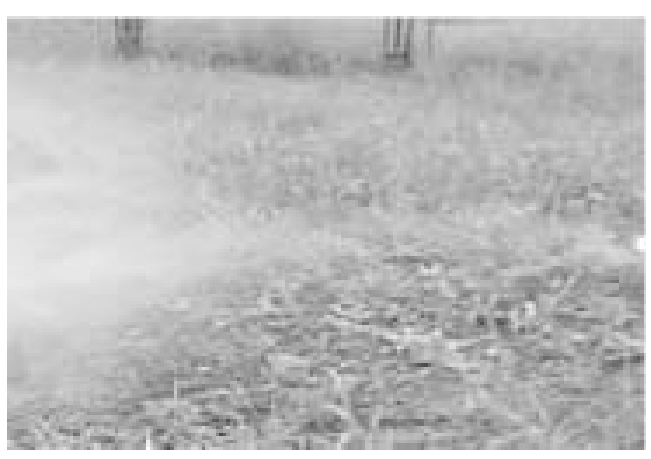

b)

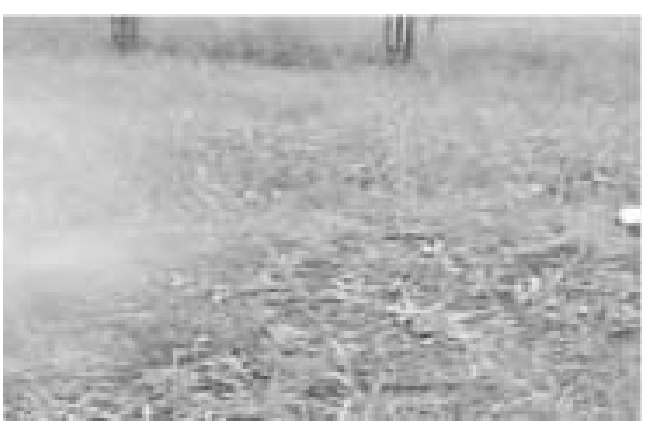

c)

Fig 11. The process of spraying water droplets: $\mathrm{a}-\mathrm{t}=0,01 \mathrm{~s} ; \mathrm{b}-\mathrm{t}=0,05 \mathrm{~s} ; \mathrm{c}-\mathrm{t}=0,10 \mathrm{~s}$

\section{Conclusions}

1. The classification of inpipe robots, their advantages and imperfections are reviewed. The schemes of the construction of the wall press walking inpipe robot with a pneumatic drive and inpipe robot with a vibratory pneumatic drive are given. 
2. When analyzing pressure and velocity variation diagrams, on the pipeline section under control at various leakage holes diameter it was identified that the larger the diameter of a leakage hole is, the greater variations of pressure and fluid flow velocity in a pipeline at leakage are. The distance from the beginning of the pipeline section under control to the leak is identified much more accurately when the diameter of a leaking hole is greater. When the diameter of a leakage hole is $10 \mathrm{~mm}$, the relative error of leak estimation is $3,575 \%$, and when the diameter of a leakage hole is $100 \mathrm{~mm}$, the relative error of the identified leak is $1,627 \%$.

The impulsive extinguishing technologies have not been completed yet and need to be further updated and tested. They allow us to use the properties of the extinguishing water more efficiently and reduce the response time.

\section{References}

1. Matuliauskas, A.; Mištinas, M.; Spruogis. B.; Ragulskis, K. Pipe crawling robots with vibratory drives. Journal of Vibroengineering, No 4 (5), 2000, p. 9-14.

2. Roh, S. G.; Choi, H. R. Strategy for Navigation Inside Pipelines with Differential-drive Inpipe Robot. In: Proceedings IEEE Int. Conf. on Robotics and Automation (ICRA 2002), 2002, p. 2575-2580.

3. Kulvietis, G.; Matuliauskas, A.; Mištinas, M.; Spruogis, B.; Ragulskis, K. Dynamic Parameter Investigation of Pipe robots. Journal of Vibroengineering, No 2 (7), 2001, p. 5558.

4. Matuliauskas, A.; Spruogis, B. Pipeline Robots with Elastic Elements. Transport. Vol XVII, No 5, Vilnius: Technika, 2002, p. 177-181.

5. Mištinas, V.; Spruogis, B. Development of Pipe Crawling robots with vibratory drives. Transport. Vol XVII, No 5, Vilnius: Technika, 2002, p. 171-176.

6. Bogdevicius, M. Identification of pipeline and fluid parameters by the method of characteristics. Transport Engineering (Transportas), 2000, Vol XV, No 3, p. 150-152 (in Russian).

7. Bajoraityte, I.; Bogdevičius, M. Dynamic processes in the main oil pipelines. Part I.: The analysis of causes of leakage occurrence in oil pipelnes and leak identification methods. Transport, 2002, Vol XVII, No 6, p. 234-240.

8. Bajoraitytè, I.; Bogdevičius, M. Dynamic processes in the main oil pipelines. Part II. : The leak identification by numerical methods. Transport, 2003, Vol XVIII, No 1, p. 4955.

9. Bogdevicius, M. Non-stationary movement of fluid in the elastic, visco-elastic, and plastic pipe. Applied Mechanics (Taikomoji mechanika), 1994, No 2, p. 117-124 (in Lithuanian).

10. Bogdevičius, M. Simulation of Hydraulic Systems of the Pump by the Method of Characteristics. Transport Engineering (Transportas). No 2(15), Vilnius: Technika, 1997, p. 30-37 (in Russian).
11. Aladjev, V.; Bogdevicius, M. Maple 6: Solution of the Mathematical, Statistical and Engineering-physical Problems (Maple 6: решение математических, статистических и инженерно-физических задач). Moscow, 2001. 824 p. (in Russian).

12. Bogdevičius, M. Simulation Interaction of Mechanical and Hydraulic System. In: Proceedings "Tenth World Congress on the Theory of Machines and Mechanisms". Oulu, Finland, 1999, p. 2110- 2115.

13. Aladjev, V.; Bogdevicius, M.; Prentkovskis, O. New Software for Mathematical Package Maple of Releases 6,7 and 8. Vilnius, 2002. 403 p.

14. Suslavičius, V.; Bogdevičius, M. Improvement of technical parameters of fire vehicles and equipment. Transport Engineering (Transportas), Vol XVIII, No 2, Vilnius: Technika, 2003, p. 89-96. 